\title{
Bimanual passive movement: functional activation and inter-regional coupling
}

\author{
Emiliano Macaluso $^{1 *}$, Andrea Cherubini ${ }^{1}$ and Umberto Sabatini ${ }^{2}$ \\ 1. Neuroimaging Laboratory, Santa Lucia Foundation, Italy \\ 2. Radiology Department, Santa Lucia Foundation, Italy \\ Edited by: Sidney A. Simon, Duke University Medical Center, USA \\ Reviewed by: Sidney A. Simon, Duke University Medical Center, USA \\ Ivan Toni, F.C. Donders Centre for Cognitive Neuroimaging, Netherlands
}

\begin{abstract}
The aim of this study was to investigate intra-regional activation and inter-regional connectivity during passive movement. During fMRI, a mechanic device was used to move the subject's index and middle fingers. We assessed four movement conditions (unimanual left/right, bimanual symmetric/asymmetric), plus Rest. A conventional intra-regional analysis identified the passive stimulation network, including motor cortex, primary and secondary somatosensory cortex, plus the cerebellum. The posterior (sensory) part of the sensory-motor activation around the central sulcus showed a significant modulation according to the symmetry of the bimanual movement, with greater activation for asymmetric compared to symmetric movements. A second set of fMRI analyses assessed condition-dependent changes of coupling between sensory-motor regions around the superior central sulcus and the rest of the brain. These analyses showed a high inter-regional covariation within the entire network activated by passive movement. However, the specific experimental conditions modulated these patterns of connectivity. Highest coupling was observed during the Rest condition, and the coupling between homologous sensory-motor regions around the left and right central sulcus was higher in bimanual than unimanual conditions. These findings demonstrate that passive movement can affect the connectivity within the sensory-motor network. We conclude that implicit detection of asymmetry during bimanual movement relies on associative somatosensory region in post-central areas, and that passive stimulation reduces the functional connectivity within the passive movement network. Our findings open the possibility to combine passive movement and inter-regional connectivity as a tool to investigate the functionality of the sensory-motor system in patients with very poor mobility.
\end{abstract}

Keywords: passive movement, bimanual, effective connectivity, fMRI

\section{INTRODUCTION}

Everyday activities often require co-ordination of the left and the right hand. The degree of symmetry (or asymmetry) of the bimanual movement can dramatically affect performance. For example, it is more difficult to produce asymmetrical sequences of finger-movements with the left and right hand, with asymmetrical movements typically yielding to a spontaneous switch to symmetrical movements (e.g., Swinnen et al., 1997). The interference of asymmetric movements is thought to reflect some limitations of the central nervous system in the simultaneous control of different actions (Pashler, 1994).

The physiological bases of these limitations are still debated. The generalized motor program model suggests that the interference observed during performance of different movements with the two hands arises because of computational limitations of a common "movement control

\footnotetext{
* Correspondence: Emiliano Macaluso, Neuroimaging Laboratory, Santa Lucia Foundation, Via Ardeatina 306, 00179 Rome, Italy. e-mail: e.macaluso@hsantalucia.it Received: 30 Aug. 2007; paper pending published: 11 0ct. 2007; accepted: 29 Nov. 2007; Published online: 30 Dec. 2007

Full citation: Frontiers in Integrative Neuroscience (2007) 1:5 doi: 10.3389/neuro.07/ 005.2007

Copyright (C) 2007 Macaluso, Cherubini and Sabatini. This is an open-access article subject to an exclusive license agreement between the authors and the Frontiers Research Foundation, which permits unrestricted use, distribution, and reproduction in any medium, provided the original authors and source are credited.
}

center" (see Schmidt, 1975). A different model (neural crosstalk) suggests that these limitations may arise because of interactions between separate control systems for the two hands/effectors (see Marteniuk and MacKenzie, 1980), thus emphasizing inter-regional dynamics during movement control (Swinnen et al., 1991; Serrien et al., 2006). Indeed, studies with split-brain patients showed that spatial assimilation effects (i.e., the tendency of performing symmetrical movements) during nonsymmetric bimanual movements are abolished when the corpus callosum is transected (Kennerley et al., 2002). These findings support the hypothesis that the flow of information between the two hemispheres plays a role during the performance of bimanual movements (Kennerley et al., 2002, see also Geffen et al., 1994).

The recent development of $\mathrm{fMRl}$ analysis techniques now allows measurement of, not only intra-regional task-related blood oxygenation level dependent (BOLD) changes, but also changes of inter-cortical coupling (connectivity). The introduction of these techniques allows the testing of hypotheses concerning network dynamics, such as those postulated by the neural crosstalk model for bimanual motor control. Several different methods can be used to investigate non-invasively interregional coupling in the human brain. These include measures of functional connectivity (assessing overall coupling between signal changes in different brain regions), and more specific effective connectivity measures that can evaluate task-related changes of interregional coupling. Effective connectivity requires the specification of a "model" concerning the interactions between regions. This may simply consist of some assumptions regarding the neuronal-BOLD coupling and 
details about the experimental design (e.g., the timing of the stimuli and the task, as in psycho-physiological interaction (PPI) analyses, Friston et al., 1997; Gitelman et al., 2003); or more complex anatomical models, comprising also specific connections between pre-specified brain regions (e.g., dynamic causal modeling (DCM), Friston et al., 2003, or structural equation modeling (SEM), e.g., Zhuang et al., 2005, see also Penny et al., 2004). It should be observed that all fMRI connectivity techniques are applied to hemodynamic BOLD data, not to neural firing data, and therefore the estimation of the connectivity parameters are driven by relatively low frequency fluctuations (approx. between 0.2 and $0.01 \mathrm{~Hz}$ ). Measures of effective connectivity have been recently employed in many sensory, motor, and cognitive domains (e.g., Dodel et al., 2005; Hampson et al., 2006; Lenartowicz and McIntosh, 2005; Mechelli et al., 2003; Rissman et al., 2004; Rowe et al., 2002). It should be noted that the more complex models of effective connectivity (e.g., DCM) permit inference about the directionality of influence between regions (e.g., area $A$ affects area $B$, rather than area $B$ affects area $A$ ), but can be biased by the specification of the anatomical connection-model. On the other hand, simpler approaches (e.g., PPIs) do not permit any directional inference (they just measure task-related changes of coupling), but do not require any a priori specification of the underling anatomical connectivity.

Here we used PPIs to investigate task-related inter-regional coupling within the sensory-motor system. We measured inter-regional coupling during passive rather than active movement. Passive movement has been utilized in several previous neuroimaging studies, because it can provide a performance-independent paradigm to activate the sensorymotor network. This is of particular relevance for the investigation of physiological-changes associated with motor recovery after brain injury, when active motor performance is often impaired (e.g., see Cramer et al., 2002; Lee et al., 1998; Matteis et al., 2003). Previous PET and fMRI studies that investigated (intra-regional) BOLD changes during passive movement provided evidence for the activation of sensory-motor regions. These include contralateral primary sensory-motor areas (Mima et al., 1999; Weiller et al., 1996), secondary somatosensory areas (SIl; activating unilaterally, Mima et al., 1999, or bilaterally, Sahyoun et al., 2004) and the supplementary motor area (SMA) (Reddy et al., 2001; Sahyoun et al., 2004; Weiller et al., 1996). Additional activations for passive movement have been reported in the inferior parietal cortex (Weiller et al., 1996) and the cerebellum (Carel et al., 2000; Loubinoux et al., 2001; Thickbroom et al., 2003). Thus, passive movement paradigms are a suitable way to investigate-at least some aspects of - the functionality of the sensorymotor network recruited during active movements (cf. Colebatch et al., 1991; Fink et al., 1997).

The objective of the present fMRI study was to extend the description of the passive-movement network using measures of effective connectivity. We investigated inter-regional changes of coupling as a function of the level of lateralization of the passive movement (unimanual vs. bimanual); and for bimanual movements, as function of the symmetry/ asymmetry of the movement. We expected that the degree of bimanual involvement (Uni- vs. Bi-manual and/or Sym- vs. Asym) would yield to changes of inter-regional coupling, highlighting the role of network's dynamic during passive movement.

\section{MATERIALS AND METHODS Subjects}

Twelve healthy right-handed volunteers (six women, six men, mean age $=24$ years) took part in the study. All subjects gave written informed consent according to institutional guidelines (Independent Local Ethics Committee of the Santa Lucia Foundation).

\section{Passive movement device}

Subjects laid supine in the scanner with their upper arms next to the body, and the hands resting over the passive movement device. This consisted

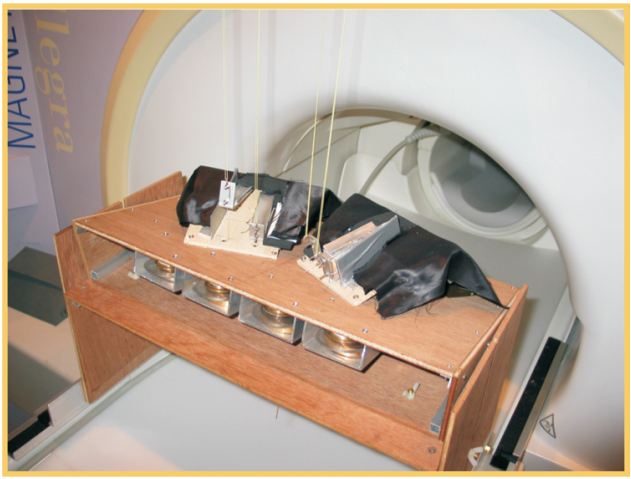

Figure 1. Device for passive movement stimulation. Picture of the MR compatible device for passive motor stimulation of the index- and middlefingers of the left and right hands.

of a system of cables connected to four levers, on which the subjects rested the index and middle fingers of each hand (see Figure 1). The device was built using non-magnetic materials (wood, plastics, and aluminum), and four electrical motors placed outside the scanner room. A computer controlled the movement of each motor independently. Passive finger-movements started with an extension (approx. 30 ) for $200 \mathrm{ms,}$ followed by a $200 \mathrm{~ms}$ phase during which the finger was returned to the resting position. Critically, all movements were operated via computer, thus reducing to a minimum the variability of the stimulation between successive trials/conditions and across subjects.

\section{Experimental conditions}

The fMRI protocol consisted of five conditions: unimanual right (UniR), unimanual left (UniL), bimanual symmetric (BiSym), bimanual asymmetric movements (BiAsym), and Rest. Each condition was blocked for 30 seconds (30 movements for each individual finger, mean frequency $=1 \mathrm{~Hz}$ ) and it was repeated four times per fMRI-run. Each subject underwent two runs. The order of presentation of the five conditions was randomized within run.

For each hand, the sequence of index-finger and middle-finger movements was random (1:1 ratio of index- and middle-finger movements), and different for each block, fMRI-run and subject. In the unimanual conditions the sequence was presented to a single hand, and only one finger was moved at any given time. In the BiSym condition the same sequence was presented symmetrically to both hands, with either the index fingers or the middle fingers set in motion simultaneously. In the BiAsym condition the four fingers moved independently, randomizing both the index/middle-finger sequence and the onset of the movement of each finger. Thus, BiSym and BiAsym conditions differed both because of the number of fingers that could move at any one time (two in BiSym, but up to four in BiAsym) and because of the relative timings of the movementonsets. This allowed us to produce highly de-correlated sequences for the two sides that would not be possible using temporally synchronous movement-onsets. Nonetheless, the total number of movements of each finger was kept constant in all conditions (30 movements, for each 30 seconds block).

Subjects were instructed to remain relaxed, and not to actively move their hands or fingers during the passive movement stimulation. Because of technical reasons, muscle activity was not measured in the MR scanner. However, the passive-movements sequences were unpredictable and rapid (finger extension in $200 \mathrm{~ms}$ ), minimizing the possibility of voluntary movements during the stimulation. In the Rest condition, subjects were asked to relax and not to move or mentally simulate finger-movements. 


\section{MR scanning and data analysis}

Functional images were acquired with an Allegra scanner operating at 3T (Siemens, Erlangen, Germany). BOLD contrast was obtained using echo-planar $\mathrm{T}^{*}$ weighted imaging (38 slices, in-plane resolution $=$ $3 \times 3 \mathrm{~mm}$, slice thickness $=2.5 \mathrm{~mm}$, inter-slice distance $=1.25 \mathrm{~mm}$, $\mathrm{TR}=2.47$ seconds, $\mathrm{TE}=30 \mathrm{~ms}$ ).

Functional MRI data were analyzed using the SPM2 (www.fil.ion. ucl.ac.uk/spm). The first four volumes of each fMRI-run were discarded to allow the signal intensities to reach equilibrium, leaving 246 volumes for each run. A rigid-body transformation (realignment) and slice-timing served to correct for head movement and slice-acquisition delays. To facilitate inter-subject (group) analyses, all images were normalized to the MNI-space using the mean of the functional volumes and then smoothed with a Gaussian filter of $8 \mathrm{~mm}$ full width at half maximum (FWHM).

We performed two types of analysis of the fMRI time series: a standard intra-regional analysis and a set of inter-regional analyses of effective connectivity. For the standard analysis, we estimated the effects associated with the experimental design on a voxel-by-voxel basis, using the general linear model. The five conditions were coded as 30 seconds epoch-blocks convolved with the SPM2 hemodynamic response function (HRF). For each subject, linear compounds (contrasts) were used to estimate the effect of each condition across the two fMRI-runs. This led to the creation of five contrast-images per subject (including the Rest condition). These contrast-images underwent a within-subject ANOVA, for group-level statistical inference. Finally, contrasts were used to compare these effects, now using between-subjects variance (rather than between scans). In the group analysis, we tested for: (a) the overall effect of passive movement versus Rest; (b) the effect of Bimanual versus Unimanual movements; and (c) the effect of movement symmetry (BiSym minus BiAsym; and BiAsym minus BiSym). Correction for non-sphericity was used to account for possible differences in error variance across conditions and any non-independent error terms for the repeated measures (Friston et al., 2002). The statistical threshold was set to $p$-corr $=0.05$, corrected for multiple comparisons (Familywise error, at voxel-level). For contrast "a" we considered the whole brain as the volume of interest, for contrast " $b$ " and " $c$ " the volume of interest was defined as all voxels activated in contrast "a", i.e., the regions showing an effect of passive movement (small volume correction, Worsley et al., 1996).

The second set of analyses tested for condition-dependent changes of coupling between sensory-motor regions around the superior central sulcus and the rest of the brain. This was done using PPIs (Friston et al., 1997), which seeks to explain activity in a brain region as the interaction between an experimental manipulation (psychological factor) and the activity in another "seed"-region (physiological factor). Here we considered how the connectivity within the sensory-motor network changed as a function of condition (UniL, UniR, BiSym, BiAsym, Rest). In two separate analyses, we used as the seed-region the left and right pericentral areas that activated during passive movement (see Figure 2). For each seed-region, we averaged activity within a 6-mm-radius sphere, centered at the peak-activation of the contrast "all movement conditions versus Rest" in the standard intra-regional group analysis (see Table 1). The size of the seed-region was comparable to the smoothness of the data, which optimized group-level statistical analyses. For each subject and each seed-region, a multiple regression analysis modeled the effects of condition, the activity of the seed-region, and the critical region-bycondition interaction (i.e., the PPI). It is worth noting that the interaction effect is orthogonal to the two main effects, and that the inclusion of the two main effects in the regression model ensures that any change of coupling associated with the PPI cannot be explained by the two main effects (e.g., increased or decreased activation for one of the movement condition). Contrast-images representing the coupling of the seed-region with the rest of the brain for each subject and each condition underwent a group-level ANOVA. Within these ANOVAs, specific contrasts compared

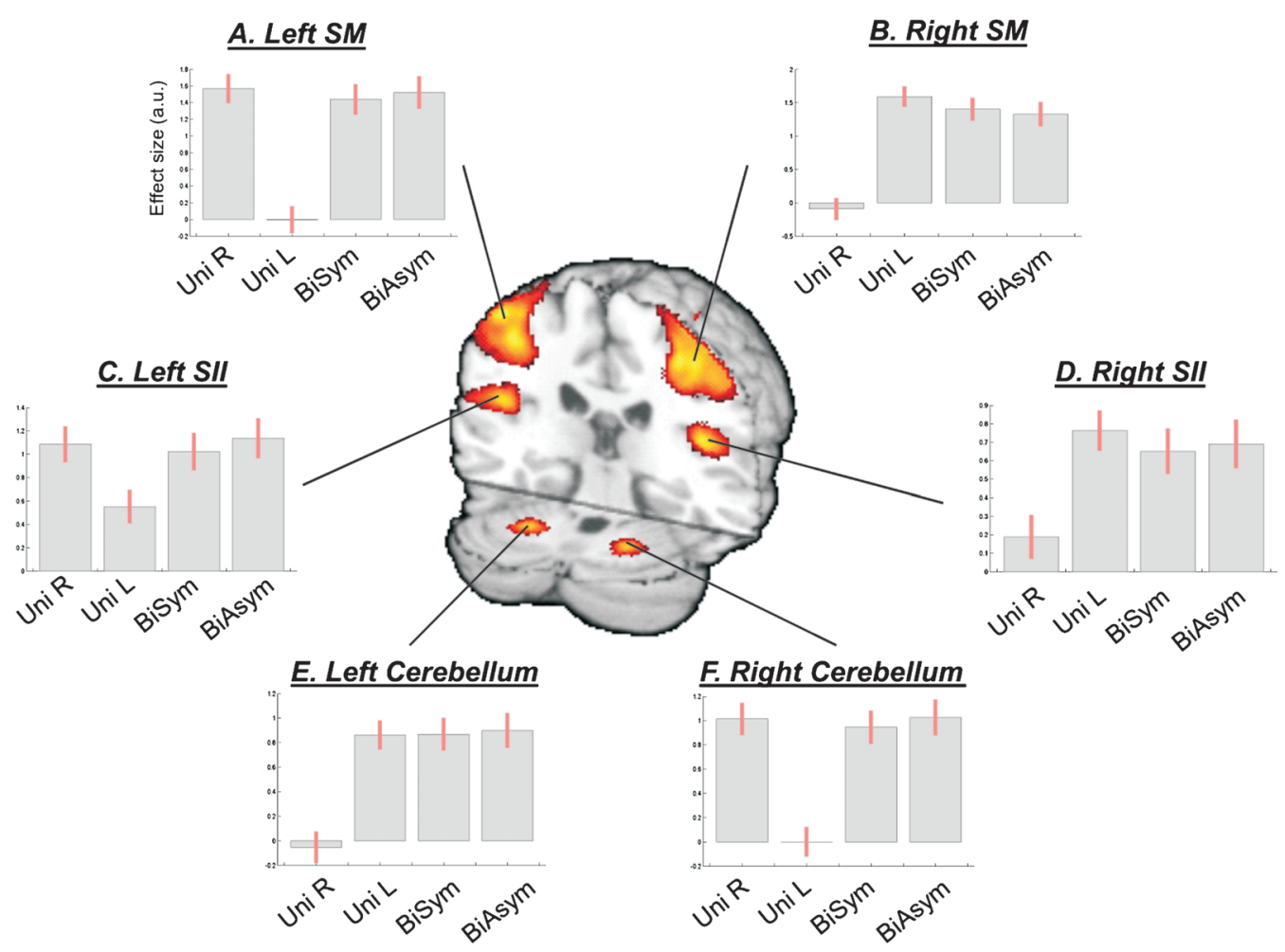

Figure 2. Network of brain areas activated during passive movement. Passive movement activated sensory-motor (SM) regions around the central sulcus contralateral to the stimulated hand, secondary somatosensory areas (SII) in the parietal operculum, and ipsilateral cerebellum. The SMA also activated for all four movement conditions (not shown, see Table 1). The signal plots display the level of activation for each movement condition compared with Rest. Effect sizes are in arbitrary units (a.u.), $\pm 90 \%$ confidence intervals. 
Table 1. All movement conditions versus Rest.

\begin{tabular}{|c|c|c|c|c|c|}
\hline & \multicolumn{3}{|c|}{ Coordinates $(x, y, z)$} & $T$-Value & $p$-corr \\
\hline \multicolumn{6}{|c|}{ Peri-central gyrus } \\
\hline $\mathrm{L}$ & -46 & -22 & 56 & 12.35 & $<0.001$ \\
\hline $\mathrm{R}$ & 50 & -22 & 56 & 12.27 & $<0.001$ \\
\hline \multicolumn{6}{|c|}{ SMA } \\
\hline $\mathrm{L}$ & -8 & -8 & 52 & 6.04 & 0.009 \\
\hline $\mathrm{R}$ & 6 & -4 & 52 & 5.96 & 0.011 \\
\hline \multicolumn{6}{|l|}{ SII } \\
\hline $\mathrm{L}$ & -48 & -22 & 16 & 11.70 & $<0.001$ \\
\hline $\mathrm{R}$ & 46 & -22 & 16 & 9.15 & $<0.001$ \\
\hline \multicolumn{6}{|c|}{ Cerebellum } \\
\hline $\mathrm{L}$ & -24 & -52 & -30 & 9.56 & $<0.001$ \\
\hline $\mathrm{R}$ & 24 & -52 & -30 & 10.72 & $<0.001$ \\
\hline
\end{tabular}

Anatomical location and statistical scores for the regions that activated during passive movement stimulation versus Rest. p-values are corrected for multiple comparisons, considering the whole brain as volume of interest. L/R, Left/Right hemisphere.

inter-regional coupling as a function of condition. We tested for changes of coupling associated with: (a) the presence/absence of movement; (b) the lateralization of the movement (unimanual/bimanual); and (c) the degree of movement symmetry during bimanual stimulation (symmetric/ asymmetric). Statistical thresholds were set to $p$-corr $=0.05$ (FWE, at voxel-level). For these analyses, the volume of interest was defined using the main effect of "all movement conditions versus Rest," as revealed in the standard intra-regional analysis (see Figure 2).

\section{RESULTS}

\section{Intra-regional activation analysis}

Passive movement conditions versus Rest. First, we highlighted the network responding to the passive-movement stimuli, comparing all movement conditions versus Rest. This revealed activation of motor and SMAs, parietal, and cerebellar regions (Figure 2). Around the central sulcus, a cluster of activation extended from the superior pre-central gyrus, posteriorly to the post-central gyrus, and regions around the anterior intraparietal sulcus (see also Figure 3, in red). This cluster included the primary sensory (SI) and primary motor (M1) areas (SMregions). In addition, significant activation was found bilaterally in the parietal operculum (secondary somatosensory area, SII), and in the cerebellum. The SM-regions around the central sulcus responded to stimulation of the contralateral hand and to both bimanual conditions (i.e., to all conditions comprising movements of the contralateral hand; see signal plots, Figures 2A and 2B). The secondary somatosensory cortex responded bilaterally to all movement conditions, but with greater activity for stimulation of the contralateral than ipsilateral hand (see Figures 2C and 2D). In contrast, the cerebellum activated for all conditions involving movement of the ipsilateral hand: i.e., UniL and both bimanual conditions, in the left hemisphere; and UniR plus bimanual, in the right hemisphere (see Figures 2E and 2F). At a lower statistical threshold ( $p$-uncorr $=$ 0.001), the same comparison revealed also activation in the ventralposterior thalamus. As for the SM-regions, activity was lateralized to the thalamus contralateral to the moving hand. Accordingly, the direct comparison of UniL $>$ UniR activated the right thalamus $(x, y, z=$ $18,-20,4 ; \quad T$-value $=4.88)$, while UniR $>$ UniL activated the left thalamus $(x, y, z=-14,-20,-2 ; T$-value $=5.10)$.

We further characterized the localization of the SM-activation using probability maps (Eickhoff et al., 2005). This revealed the following distribution of activated voxels. Left hemisphere: $25.6 \%$ in Area 2; $18.3 \%$ in Area $1 ; 17.1 \%$ in Area 6; 9.4\% in Area 3b; $8.5 \%$ in Area $4 p ; 6.1 \%$ in Area 4a. Right hemisphere: $20.0 \%$ in Area 2; $17.2 \%$ in Area 1; $15.7 \%$ in Area $6 ; 10.8 \%$ in Area 3b; $9.6 \%$ in Area $4 p ; 7.0 \%$ in Area $4 a$.

Bimanual versus unimanual movement. Next, we assessed whether simultaneous stimulation of both hands (bimanual conditions) would
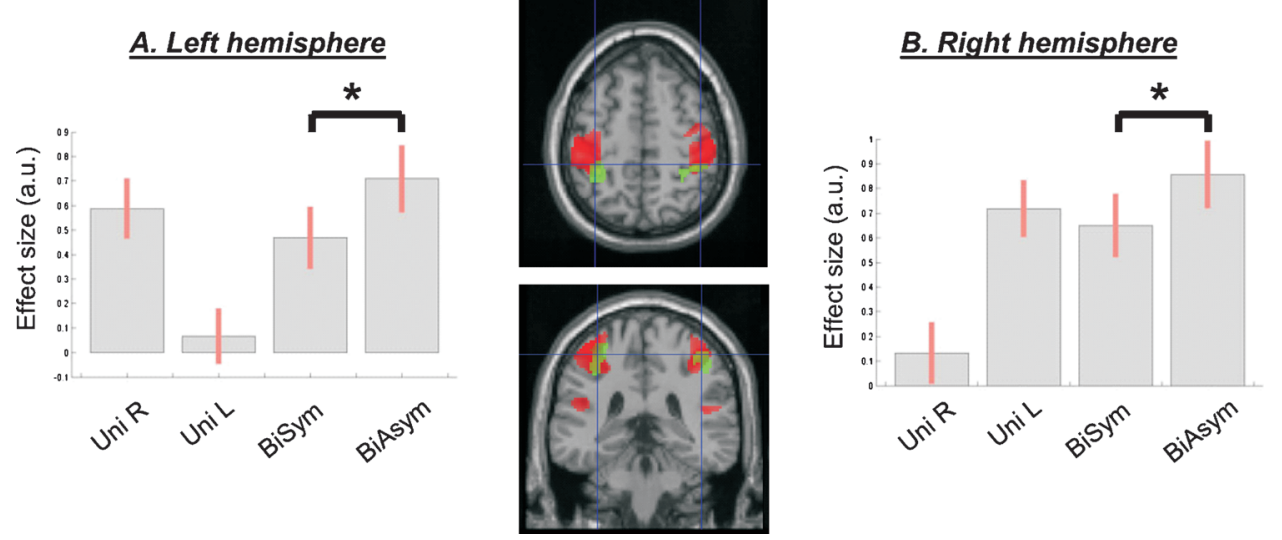

Figure 3. Intra-regional activation for asymmetric bimanual movements. A region at the interception of the anterior intraparietal sulcus and the post-central gyrus (in green) showed greater activation for asymmetric compared to symmetric bimanual passive movements (compare bar 4 vs. 3, in each plot). The figure also displays the overall effect of passive movement (in red, see also Figure 2), demonstrating the posterior localization of the a-/symmetry effect. The signal plots show the level of activation for each movement condition compared with Rest. Effect sizes are in arbitrary units (a.u.), $\pm 90 \%$ confidence intervals. 
trigger some specific activation (or modulation of activity), over and above any effect observed during passive movement of one or the other hand (unimanual conditions). No region showed a significant enhancement of activity during bimanual stimulation. However, in the secondary somatosensory cortex the BOLD response was non-linear, with activity in the bimanual conditions smaller than the sum of activity in the two unimanual conditions. This can be observed in the signal plots of Figure 2, panels $C$ and $D$ : activity in the two bimanual conditions (two rightmost bars) was similar to the activity observed during passive movement of the contralateral hand, rather than the sum of activity for UniL + UniR (two leftmost bars in the signal plots Figures $2 \mathrm{C}$ and 2D).

A-/symmetry of the bimanual passive movement. One of the central aims of this study was to compare conditions of passive movement, entailing different levels of a-/symmetry for the two hands. Accordingly, we contrasted directly the two bimanual conditions (BiSym and BiAsym). This revealed that activity in posterior part of the post-central gyrus and surrounding anterior intraparietal regions increased in the asymmetric bimanual condition (BiAsym) when different sequences of index/middlefinger movements were delivered to the left and the right hand. In the left hemisphere the activation-peak was located at $x, y, z=-34,-42,56$ $(T=5.30 ; p$-corr $=0.002)$; and in the right hemisphere it was at $x, y, z=40,-38,56(T=4.53 ; p$-corr $=0.019)$. Figure 3 shows the anatomical location (in green) and the signal plot for these regions. The same figure also displays the location/extent of the main effect of movement versus Rest (in red). These overlapping-projections reveal that the area modulated by the a-/symmetry of the bimanual movement was located in the more posterior, "sensory" part of the large sensory-motor cluster. The signal plots show the contralateral responses in this area during unimanual conditions (compare bar 1 and bar 2), and critically a greater activation for BiAsym (bar 4) compared with BiSym (bar 3).

Using probability maps (Eickhoff et al., 2005), we localized the activation related to the BiAsym condition, as follows: Left hemisphere: $40.4 \%$ in Area 2; $18.2 \%$ in Area 3b; $12.0 \%$ in Area $4 p ; 8.5 \%$ in Area 3a; $1.5 \%$ in Area $6 ; 1.3 \%$ in Area 4a. Right hemisphere: $37.6 \%$ in Area 2; $25.7 \%$ in Area 3b; $10.3 \%$ in Area 4a; $5.7 \%$ in Area 3a; $1.4 \%$ in Area 1.

\section{Effective connectivity: changes of inter-regional coupling}

We performed two PPI analyses (Friston et al., 1997) to test for changes of coupling of the left and right SM-regions, as a function of movement condition. For each hemisphere, we estimated the coupling of these areas with the rest of the brain, separately for the five experimental conditions (UniL, UniR, BiSym, BiAsym, and Rest).

Example of inter-regional coupling in a single subject. Before turning to the group analyses, we briefly illustrate the estimation of the connectivity parameters for a single subject. Figure 4 shows the results of the PPI analysis seeded in the left SM-region. This area showed coupling with neighboring SM-regions in the left hemisphere and with the homologous SM-region in the right hemisphere (see Figure 4A). Figure 4B shows the BOLD signal in the two SM-regions plotted against each other, separately for the five conditions. The coupling between the two regions was highest in the Rest condition (in blue), intermediate in the two bimanual conditions (in red and black), and lowest for the two unimanual conditions (in green and magenta). The amount of coupling is captured by the slope of the regression line. This critical parameter was estimated for each condition and each subject, and used for group-level statistical inference about condition-dependent connectivity (see next section).

Group analyses. The group analyses revealed that there was a high level of coupling between peri-central SM-cortex and all regions activated during passive movement. Figure 5 shows a $3 \mathrm{D}$ rendering of the regions where the coupling values were different from zeros ( $F$-statistics). $T$-tests revealed maximal coupling during the Rest condition, compared with the four passive movement conditions (see Table 2 for details). Thus, overall the passive movement stimulation decreased the functional connectivity within the sensory-motor network.

However, direct comparisons between the four passive movement conditions revealed that the functional coupling between the SM-regions in the left and the right hemispheres was higher during bimanual than unimanual conditions. The change of coupling from the left to the right SM was significant at a corrected level $(x, y, z=36,-28,62 ; T=5.52$, $p$-corr $=0.001)$. An analogous effect was found for the seed in the left $\mathrm{SM}$, even though this did not reach full statistical significance after correction for multiple comparison $(x, y, z=-40,-22,54 ; T=3.42$, $p$-uncorr $<0.001)$. Figure 6 shows the anatomical location of these condition-dependent effects, and the level of coupling between the two SM-regions for each experimental condition (Figures 6A and 6B). During bimanual stimulation, the right SM showed increased coupling with the left SM (in green, on the transverse section in Figure 6) and the left SM showed increased coupling with the right SM-region (in magenta). Maximal coupling was observed during the Rest condition (see bar 1 in Figure 6 plots, and cf. also Figure 5), but the coupling parameters were higher for bimanual than unimanual conditions (compare bars 4 and 5 vs. bars 2 and 3). Thus, the specific type of passive movement affected the level covariation of the BOLD signal between homologous SM-regions in the two hemispheres.

Next, we tested whether the a-/symmetry of the bimanual movement affected the coupling of the left/right SM-regions. Unexpectedly, this did not reveal any significant effect on inter-regional connectivity. Since the asymmetry of the bimanual movement modulated intra-regional activity

\section{A. Statistical map}

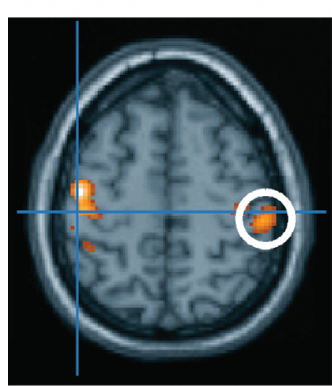

$$
z=56
$$

\section{B. leftright SM co-variation}

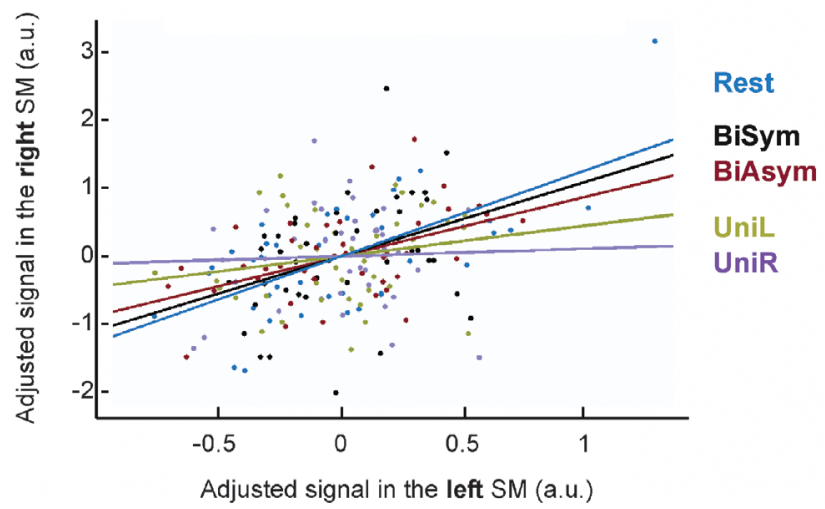

Figure 4. Example of connectivity results for a single subject. Results of the connectivity analyses seeded in the left SM-region. Panel A shows a projection of the F-map on a transverse anatomical section, revealing a covariation between the signal in the left SM-region (blue cross) and the homologous SM-region in the right hemisphere (white circle). Panel B illustrates the coupling between the two regions, as a function of condition. 


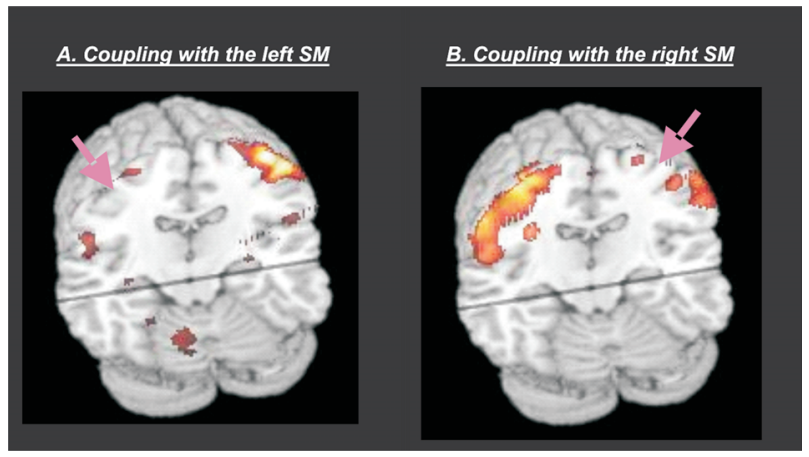

Figure 5. Group results of the connectivity analyses. The figure shows a $3 D$ rendering of the F-statistics testing for PPIs with the left SM-region (panel A) and the right SM-region (panel B). The pink arrows illustrate the approximate location of the seed-region that was used in the two PPI analyses. The results revealed high inter-regional coupling within entire SM-network (homologous SM-region in the opposite hemisphere, SII in the parietal operculum, cerebellum, and SMA). Additional t-tests revealed that the coupling was maximal in the Rest condition and that it decreased during passive movement stimulation (see also Table 2).

only in the posterior part of the SM-cluster (see Figure 3, in green), we carried out additional PPI-analyses, now using these posterior clusters as the seed-regions. These analyses did not reveal any significant effect of movement a-/symmetry on coupling strength. Finally, to test the hypothesis that movement asymmetry may affect the connectivity of high-level movement control centers; we used the activated SMA regions (cf. Table 1) as the seeds for further analyses of connectivity. Again we tested for changes of coupling depending on the a-/symmetry of the bimanual passive movement, but we did not find any significant effect. Thus, the level of bimanual a-/symmetry modulated intra-regional activation (cf. Figure 3, in green), but not the connectivity of the SM-network.

\section{DISCUSSION}

In this study, we investigated functional activation and inter-regional coupling during presentation of sequences of passive movements to the index- and middle-fingers. We presented unimanual sequences, symmetric bimanual or asymmetric bimanual sequences. A standard intra-regional analysis found that passive stimulation activated sensorymotor areas around the superior central sulcus (SM-regions), secondary somatosensory cortex in the parietal operculum (SII), SMA and the cerebellum (plus the thalamus at a lower statistical threshold). Analyses of inter-regional coupling revealed that the SM-regions displayed high levels of covariation with the other areas during Rest, and that this covariation diminished during passive movement. Furthermore, the specific type of movement affected the coupling between homologous SM-regions in the two hemispheres, with greater coupling during bimanual than unimanual conditions. The degree of a-/symmetry of the bimanual movement did not influence significantly inter-regional coupling, but a region in the postcentral gyrus showed greater intra-regional activation for asymmetric compared with symmetric movements.

Several previous studies examined brain activity associated with passive movement (e.g., Lee et al., 1998; Mima et al., 1999; Reddy et al., 2001; Thickbroom et al., 2003; Weiller et al., 1996). While passive movement is a form of sensory stimulation and it causes a predominantly proprioceptive input, it can be useful in assessment of the functionality of the sensory-motor network in patients with little mobility (e.g., Cramer et al., 2002; Jang et al., 2004; Matteis et al., 2003). Studies that used passive movement and active motor tasks in within-subject designs found largely overlapping patterns of activation, often with greater activation for active than passive tasks (Mima et al., 1999; Sahyoun et al., 2004, but see Weiller et al., 1996, who found similar activation in contralateral M1 in the two tasks). Reddy et al., (2001) reported a significant posterior shift of contralateral sensory-motor activation in passive compared with active finger-movement, possibly related to a greater involvement of the somatosensory cortex in the former condition. Here we did not directly compare active and passive movements, but we found a pattern of activation consistent with an engagement of large portions of the sensory-motor network. This included SM-regions around the superior central sulcus encompassing activation of the pre-central sulcus, likely to correspond to the primary motor cortex. The unimanual conditions demonstrated that the peri-central activation was fully lateralized to the hemisphere contralateral to the stimulated hand (cf. Figures 2A and 2B). A similar pattern of lateralization was found in the thalamus. Instead, higher-order somatosensory areas in the parietal operculum activated both for contralateral and for ipsilateral movements (see Figures 2C and 2D). This is consistent with the view that early somatosensory representations are lateralized, while later representations are bilateral (Coghill et al., 1994; Ruben et al., 2001; Simoes and Hari, 1999).

Table 2. Decreased coupling during passive movement versus Rest.

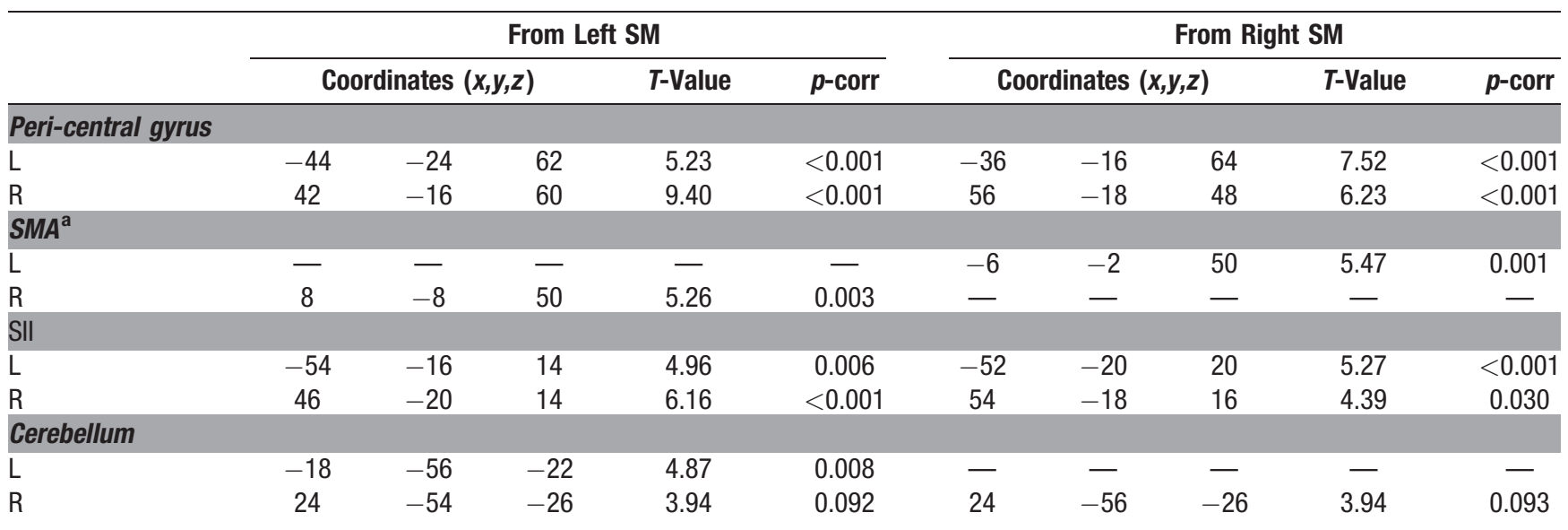

Anatomical location and statistical scores for the regions where coupling with the left SM-region (left side of the table) and the right SM-region (on the right side of the table) decreased during passive movement compared with coupling at Rest.p-values are corrected for multiple comparisons, considering as the volume of interest at voxels that activated during passive movement stimulation in the standard intra-regional analysis (cf. Figure 2). ${ }^{a}$ For the SMA, these PPI analyses revealed a single peak, but the clusters extended to both hemispheres. L/R, Left/Right hemisphere. 
A. Right SM $\rightarrow$ Left SM

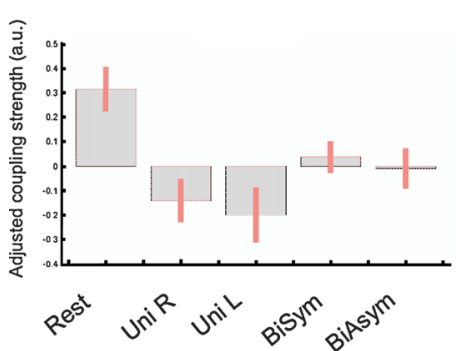

B. Left SM $\rightarrow$ Right SM

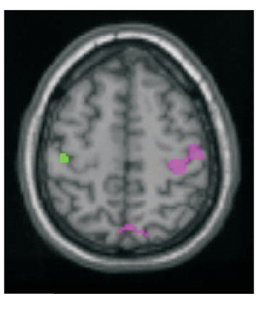

$z=56$

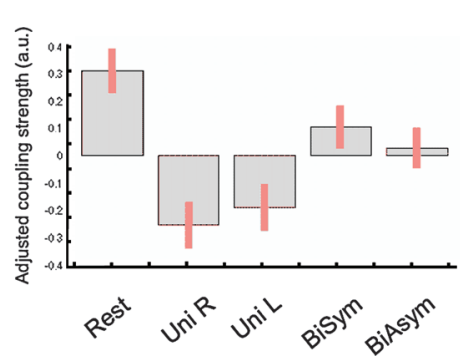

Figure 6. Inter-hemispheric connectivity between the two SM-regions. Direct comparisons between passive movement conditions demonstrated that the coupling between the left and the right SM-regions was higher during bimanual than unimanual conditions. The central panel shows the anatomical localization of the coupling effects (magenta, PPI seeded in the left SM and revealing coupling with the right SM; green, PPI seeded in the right SM and revealing coupling with the left SM). The signal plots display the coupling parameters for each condition (panel A: parameters estimates in the left SM; panel B: parameters in the right SM; in arbitrary units, $\pm 90 \%$ confidence intervals). The highest connectivity was observed during Rest (bar 1; cf. also Table 2), but coupling between SM-regions was also greater during bimanual than unimanual conditions (compare bars 4 and $5 \mathrm{vs.} \mathrm{bars} 2$ and 3). Note that the coupling parameters are mean-adjusted (mean equal zero), the actual values of the coupling parameters were 1.27, 0.80, 0.68, 0.99, 0.92, for the left SM-region; and 1.33, 0.77, 0.83, 1.12, 1.05, for the right SM-region.

Consistent with crossing projections, the cerebellar responses were lateralized to the ipsilateral hemisphere (see Figures 2D and 2E; Bushara et al., 2001; Lotze et al., 1999).

Together with the standard analysis of intra-regional activation, here we also used a simple model of effective connectivity (PPIs) to investigate condition-dependent inter-regional coupling within the passive movement network. The issue of inter-regional coupling has been previously addressed in the context of active motor tasks (e.g., Solodkin et al., 2004; Sun et al., 2004, see also Gerloff and Andres, 2002; Pollok et al., 2007, for a related EEG and MEG approaches). One of the most consistent and striking findings is that even at Rest (i.e., when subjects do not move) there is a high connectivity between the different regions of the sensorymotor network (e.g., see Biswal et al., 1995; Jiang et al., 2004; Morgan and Price, 2004; Peltier et al., 2005; Sun et al., 2004). This led to the proposal that patterns of connectivity at Rest could actually be useful to identify the network of cortical regions involved in motor execution and planning (Biswal et al., 1995; Johansen-Berg et al., 2004). Not surprisingly, also here when seeding the PPI-analyses in the SM-regions around the superior central sulcus, we found high levels of coupling with areas associated with sensory-motor processing, during the Rest condition (homologous region in the opposite hemisphere, parietal operculum, SMA, and cerebellum; see Table 2). The presence of pattern of functional connectivity during Rest is not unique to the motor system. These have been reported in the visual system (Nir et al., 2006), within the dorsal and ventral attention systems (Fox et al., 2006), and between regions that typically de-activate during task performance (Greicius et al., 2003, the so-called "default mode network," Raichle et al., 2001). Recently, Damoiseaux and colleagues (2006) were able to identify several distinct networks showing coherent spatio-temporal patterns of fluctuation, using tensor probabilistic independent component analysis. The Authors associated each network with specific functions, such as visual processing (lateral and superior occipital regions), auditory processing (superior temporal gyrus and insula), executive control and working memory (prefrontal, superior parietal, anterior and posterior cingulate), as well as sensory-motor functions (pre- and post-central gyri).

These patterns of connectivity at Rest are likely to reflect the underlying structural connection between different functional systems in the brain (e.g., see Johansen-Berg et al., 2004), but the consequences of task performance on functional connectivity are still poorly understood (but see Hampson et al., 2006; Lowe et al., 1998). Spontaneous fluctuations within cortico-thalamic loops may provide a possible mechanism mediating high inter-regional coupling at Rest. These influences would diminish upon presentation of specific sensory inputs that activate selective thalamo-cortical pathways, resulting in a reduction of inter-regional cortical coupling (see Nir et al., 2006). Concerning the sensory-motor network, previous studies pointed to a reduction of the connectivity within the SM network during active motor performance (e.g., Morgan and Price, 2004, and Stancák and Pfurtscheller, 1996, for local desynchronization measured with EEG). However, motor performance has also been found to positively modulate the level of coupling within the SM network. For example, Jiang and colleagues (2004) reported increased coupling between left M1, left pre-motor cortex, and left superior parietal cortex during performance of motor sequences with the right hand, compared with Rest. Condition-dependent coupling within the SM-system has been examined extensively using EEG measures of coherence (e.g., Andres et al., 1999; Gerloff et al., 1998; Gerloff and Andres, 2002; Serrien and Brown, 2004). These studies revealed changes of inter-hemispheric coupling as a function of specific task components, for example showing increased coupling in the early phases of bimanual learning (Andres et al., 1999), or for internally versus externally paced unimanual movements (Gerloff et al., 1998, see also Serrien et al., 2006 for review).

Using fMRI, here we found increased coupling between left and right SM-regions, when subjects were presented with bimanual passive movement sequences, compared with unimanual sequences (see Figure 6). It should be noted that our analysis procedures separated any inter-regional coupling from mere intra-regional activation, estimating both effects within the same general linear model (see Section 2; and Morgan and Price, 2004, for related issues). Hence, changes of coupling between left and right SM-regions cannot be explained by a differential activation of these regions depending on the lateralization of the passive movement (bimanual vs. unimanual). Instead, increased coupling during bimanual movements may relate to an increased level of neuronal synchronization between the two hemispheres, in the presence of a bilateral input. Increased synchronization has been linked to the integration of information between anatomically segregated regions (e.g., Varela et al., 2001, plus Brown and Marsden, 2001; Hummel and Gerloff, 2006, for examples concerning motor control). In the current experiment, the stimulation of homologous regions in the two hemispheres during the bimanual conditions may have triggered such integrative processes, with both cortical sites participating in the processing of related movement-sequences (see also Marsden et al., 2000). Alternatively, changes of coupling between left and right SM may arise because of differences in the level of inhibitory signals during bimanual and unimanual conditions. It might be expected that unimanual 
simulation requires greater suppression of inter-hemispheric interactions, in order to prevent mirror symmetric movements (Gerloff et al., 1998; Li et al., 2005; Rokni et al., 2003). Such inhibition could in principle reduce covariation between the two SM-regions during unimanual condition, yielding to lower coupling values in our fMRI connectivity analyses.

Somewhat surprisingly, the degree of symmetry of the bimanual passive movement (symmetric or asymmetric) did not influence the coupling-strength of the two SM-regions. On account of the neural crosstalk model (Marteniuk and MacKenzie, 1980), we expected stronger inhibitory interactions between left and right SM-cortex for asymmetric than symmetric sequences, when the two hemispheres have to process competing information. However, it should be noted that our passive stimulation paradigm did not require any active movement control. Thus, we hypothesize that-during passive stimulation-inhibition is critical when the sensory input activates one hemisphere only (unimanual conditions), but less so when both hemispheres are active and competition is reduced (i.e., similar activation-level in the left and right SM-regions, irrespective of bimanual a-/symmetry, see Figures 2A and 2B). A different situation may be expected in conditions of voluntary/active movements, when control factors will also come into play (e.g., tracking of any movement triggering cue, movement planning, monitoring of the movement outcomes). In this case, competitive interactions will entail motor commands, rather than mere activation-level in the two hemispheres, and specific movement parameters (e.g., the symmetry of the bimanual movement) may become more critical to regulate interhemispheric inhibitory processes.

An alternative view (computational limitation of one motor control center, e.g., see Schmidt, 1975) may instead predict an effect of movement asymmetry on the coupling between SM-cortex and other high-level motor areas. The role of bimanual coordination has been extensively investigated during performance of active movements (e.g., Jancke et al., 2000; Johansson et al., 2006; Sadato et al., 1997; Serrien et al., 2001, see also Pollok et al., 2007). The results point to a pivotal role of the SMA and pre-motor regions during performance of asymmetric (parallel) actions with the two hands (e.g., see Debaere et al., 2001; Sadato et al., 1997; Ullen et al., 2003). Here, we found activation of SMA in the standard intra-regional analysis (see Table 1). However, the intraregional activation of the SMA was not modulated according to the a-/symmetry of the passive movement, neither was its connectivity strength with other regions of the brain. This suggests that SMA activation during passive movement may not directly relate to the same motor functions characterizing SMA activation in active motor tasks. In active tasks, the SMA engages mostly when complex movements are required (e.g., Rao et al., 1993), and even for finger co-ordination during unimanual movements (Koeneke et al., 2004). Our passive movement paradigms did not require any movement planning and the SMA activation may relate to monitoring functions instead (Macar et al., 2006, see also Downar et al., 2001, for SMA involvement during passive perception in different sensory modalities).

Instead, here we found that the asymmetric bimanual condition increased activity in the posterior part of the SM-region. The cluster of activation that was modulated according to the a-/symmetry of the bimanual passive movement included the anterior parietal cortex and the somatosensory post-central gyrus (cf. Figure 3). The activation of primarily "sensory" areas (Coghill et al., 1994), as opposed to motor regions anterior to the central sulcus (e.g., SMA), is consistent with the sensory and proprioceptive nature of the passive movement stimulation. Accordingly, the modulation that we found here for passive movement may relate to implicit sensory discrimination, rather than competition/ interaction between multiple motor plans thought to arise during asymmetric active motor tasks (Swinnen et al., 1997). It should be noted that the activation associated with asymmetric stimulation may relate to either the number of fingers moving at any one time, or to the timing differences between the movement-onsets on the two sides. Here, we could not dissociate these two factors that jointly contributed to de- correlate the sensory input delivered to the left and right hands, in the BiAsym condition. The parietal activation included the anterior intraparietal sulcus and it extended dorsally to the anterior part of the superior parietal gyrus. This region includes area BA5 that is involved in postural processing (e.g., Graziano et al., 2000, see also Lloyd et al., 2003). Further, our current activation co-localized well with a region reported in an fMRI study of bimanual active movement (Wenderoth et al., 2004). Activity in this area, plus another region in the anterior dorsal pre-motor cortex, increased when subjects performed incompatible actions with the left and the right hand. The Authors associated the more posterior activation with inter-hemispheric integration of somatosensory information. Our findings for asymmetric passive movements may be consistent with this interpretation, suggesting the participation of this parietal region in bimanual (inter-hemispheric) "sensory" processing.

In summary, here we showed that passive motor sequences activated a network of brain areas similar to the one typically observed in active motor conditions (Colebatch et al., 1991; Fink et al., 1997). The a-/symmetry of the bimanual sequences lead to increased activation in a region posterior to the central sulcus, possibly reflecting implicit detection of the asymmetric sensory input to the two hemispheres. Analyses of inter-regional coupling revealed that passive stimulation reduces the functional connectivity within the SM-network. However, we also found that the coupling between homologous SM-regions around the left and right central sulcus was higher in bimanual than unimanual conditions, demonstrating changes of inter-hemispheric interactions as a function of passive movement condition. Future studies may employ passive movement and analyses of connectivity in patients with motor impairment, e.g., caused by a stroke, as a novel prognostic tool.

\section{CONFLICT OF INTEREST STATEMENT}

The authors declare that the research was conducted in the absence of any commercial or financial relationship that could be construed as a potential conflict of interest.

\section{ACKNOWLEDGMENTS}

This work was supported by the Istituto Superiore di Sanità (Italy). The Neuroimaging Laboratory is supported by The Italian Ministry of Health.

\section{REFERENCES}

Andres, F. G., Mima, T., Schulman, A. E., Dichgans, J., Hallett, M., and Gerloff, C. (1999). Functional coupling of human cortical sensorimotor areas during bimanual skill acquisition. Brain 122, 855-870.

Biswal, B., Yetkin, F. Z., Haughton, V. M., and Hyde, J. S. (1995). Functional connectivity in the motor cortex of resting human brain using echo-planar MRI. Magn. Reson. Med. 34, 537-541.

Brown, P., and Marsden, J. F. (2001). Cortical network resonance and motor activity in humans. Neuroscientist 7, 518-527.

Bushara, K. 0., Wheat, J. M., Khan, A., Mock, B. J., Turski, P. A., Sorenson, J., and Brooks, B. R. (2001). Multiple tactile maps in the human cerebellum. Neuroreport 12, 2483-2486.

Carel, C., Loubinoux, I., Boulanouar, K., Manelfe, C., Rascol, O., Celsis, P., and Chollet, F. (2000). Neural substrate for the effects of passive training on sensorimotor cortical representation: a study with functional magnetic resonance imaging in healthy subjects. J. Cereb. Blood Flow Metab. 20, 478-484.

Coghill, R. C., Talbot, J. D., Evans, A. C., Meyer, E., Gjedde, A., Bushnell, M. C., and Duncan, G. H. (1994). Distributed processing of pain and vibration by the human brain. J. Neurosci. 14, 4095-4108.

Colebatch, J. G., Deiber, M. P., Passingham, R. E., Friston, K. J., and Frackowiak, R. S (1991). Regional cerebral blood flow during voluntary arm and hand movements in human subjects. J. Neurophysiol. 65, 1392-1401.

Cramer, S. C., Mark, A., Barquist, K., Nhan, H., Stegbauer, K. C., Price, R., Bell, K. Odderson, I. R., Esselman, P., and Maravilla, K. R. (2002). Motor cortex activation is preserved in patients with chronic hemiplegic stroke. Ann. Neurol. 52, 607-616.

Damoiseaux, J. S., Rombouts, S. A., Barkhof, F., Scheltens, P., Stam, C. J., Smith, S. M., and Beckmann, C. F. (2006). Consistent resting-state networks across healthy subjects. Proc. Natl. Acad. Sci. USA 103, 13848-13853.

Debaere, F., Swinnen, S. P., Beatse, E., Sunaert, S., Van, H. P., and Duysens, J. (2001). Brain areas involved in interlimb coordination: a distributed network. Neuroimage 14, 947-958. 
Dodel, S., Golestani, N., Pallier, C., Elkouby, V., Le, B. D., and Poline, J. B. (2005). Condition-dependent functional connectivity: syntax networks in bilinguals. Philos. Trans. R. Soc. Lond. B. Biol. Sci. 360, 921-935.

Downar, J., Crawley, A. P., Mikulis, D. J., and Davis, K. D. (2001). The effect of task relevance on the cortical response to changes in visual and auditory stimuli: an event-related fMRI study. Neuroimage 14, 1256-1267.

Eickhoff, S. B., Stephan, K. E., Mohlberg, H., Grefkes, C., Fink, G. R., Amunts, K., and Zilles, K. (2005). A new SPM toolbox for combining probabilistic cytoarchitectonic maps and functional imaging data. Neuroimage 25, 1325-1335.

Fink, G. R., Frackowiak, R. S., Pietrzyk, U., and Passingham, R. E. (1997). Multiple nonprimary motor areas in the human cortex. J. Neurophysiol. 77, 21642174.

Fox, M. D., Corbetta, M., Snyder, A. Z., Vincent, J. L., and Raichle, M. E. (2006). Spontaneous neuronal activity distinguishes human dorsal and ventral attention systems. Proc. Natl. Acad. Sci. USA 103, 10046-10051.

Friston, K. J., Buechel, C., Fink, G. R., Morris, J., Rolls, E., and Dolan, R. J. (1997). Psychophysiological and modulatory interactions in neuroimaging. Neuroimage 6 , 218-229.

Friston, K. J., Glaser, D. E., Henson, R. N., Kiebel, S., Phillips, C., and Ashburner, J. (2002). Classical and Bayesian inference in neuroimaging: applications. Neuroimage 16, 484-512.

Friston, K. J., Harrison, L., and Penny, W. (2003). Dynamic causal modelling. Neuroimage 19, 1273-1302.

Geffen, G. M., Jones, D. L., and Geffen, L. B. (1994). Interhemispheric control of manual motor activity. Behav. Brain Res. 64, 131-140.

Gerloff, C., and Andres, F. G. (2002). Bimanual coordination and interhemispheric interaction. Acta Psychol. (Amst) 110, 161-186.

Gerloff, C., Richard, J., Hadley, J., Schulman, A. E., Honda, M., and Hallett, M. (1998) Functional coupling and regional activation of human cortical motor areas during simple, internally paced and externally paced finger movements. Brain 121, 15131531.

Gitelman, D. R., Penny, W. D., Ashburner, J., and Friston, K. J. (2003). Modeling regional and psychophysiologic interactions in fMRI: the importance of hemodynamic deconvolution. Neuroimage 19, 200-207.

Graziano, M. S., Cooke, D. F., and Taylor, C. S. (2000). Coding the location of the arm by sight. Science 290, 1782-1786.

Greicius, M. D., Krasnow, B., Reiss, A. L., and Menon, V. (2003). Functional connectivity in the resting brain: a network analysis of the default mode hypothesis. Proc. Natl. Acad. Sci. USA 100, 253-258.

Hampson, M., Driesen, N. R., Skudlarski, P., Gore, J. C., and Constable, R. T. (2006). Brain connectivity related to working memory performance. J. Neurosci. 26, 1333813343.

Hummel, F. C., and Gerloff, C. (2006). Interregional long-range and short-range synchrony: a basis for complex sensorimotor processing. Prog. Brain Res. 159, 223236

Jancke, L., Peters, M., Himmelbach, M., Nosselt, T., Shah, J., and Steinmetz, H. (2000). fMRI study of bimanual coordination. Neuropsychologia 38, 164-174.

Jang, S. H., Kim, Y. H., Chang, Y., Han, B. S., Byun, W. M., and Chang, C. H. (2004). The predictive value of cortical activation by passive movement for motor recovery in stroke patients. Restor. Neurol. Neurosci. 22, 59-63.

Jiang, T., He, Y., Zang, Y., and Weng, X. (2004). Modulation of functional connectivity during the resting state and the motor task. Hum. Brain Mapp. 22, $63-71$.

Johansen-Berg, H., Behrens, T. E., Robson, M. D., Drobnjak, I., Rushworth, M. F., Brady, J. M., Smith, S. M., Higham, D. J., and Matthews, P. M. (2004). Changes in connectivity profiles define functionally distinct regions in human medial frontal cortex. Proc. Natl. Acad. Sci. USA 101, 13335-13340.

Johansson, R. S., Theorin, A., Westling, G., Andersson, M., Ohki, Y., and Nyberg, L. (2006). How a lateralized brain supports symmetrical bimanual tasks. PLoS Biol. 4 158

Kennerley, S. W., Diedrichsen, J., Hazeltine, E., Semjen, A., and Ivry, R. B. (2002). Callosotomy patients exhibit temporal uncoupling during continuous bimanual movements. Nat. Neurosci. 5, 376-381.

Koeneke, S., Lutz, K., Wüstenberg, T., Jäncke, L. (2004). Bimanual versus unimanual coordination: what makes the difference?. Neuroimage 22, 1336-1350.

Lee, C. C., Jack, C. R., Jr., RiedererS. J. (1998). Mapping of the central sulcus with functional MR: active versus passive activation tasks. Am. J. Neuroradiol. 19, 847852 .

Lenartowicz, A., and Mclntosh, A. R. (2005). The role of anterior cingulate cortex in working memory is shaped by functional connectivity. J. Cogn. Neurosci. 17, 10261042.

Li, Y., Levin, O., Forner-Cordero, A., and Swinnen, S. P. (2005). Interactions between interlimb and intralimb coordination during the performance of bimanual multijoint movements. Exp. Brain Res. 163, 515-526.

Lloyd, D. M., Shore, D. I., Spence, C., and Calvert, G. A. (2003). Multisensory representation of limb position in human premotor cortex. Nat. Neurosci. 6, 1718.

Lotze, M., Montoya, P., Erb, M., Hulsmann, E., Flor, H., Klose, U., Birbaumer, N., and Grodd, W. (1999). Activation of cortical and cerebellar motor areas during executed and imagined hand movements: an fMRI study. J. Cogn. Neurosci. 11, 491-501.

Loubinoux, I., Carel, C., Alary, F., Boulanouar, K., Viallard, G., Manelfe, C., Rascol, O., Celsis, P., and Chollet, F. (2001). Within-session and between-session reproducibility of cerebral sensorimotor activation: a test-retest effect evidenced with functional magnetic resonance imaging. J. Cereb. Blood Flow Metab. 21, 592607

Lowe, M. J., Mock, B. J., and Sorenson, J. A. (1998). Functional connectivity in single and multislice echoplanar imaging using resting-state fluctuations. Neuroimage 7, 119132

Macar, F., Coull, J., and Vidal, F. (2006). The supplementary motor area in motor and perceptual time processing: fMRI studies. Cogn. Process 7, 89-94.

Marsden, J. F., Werhahn, K. J., Ashby, P., Rothwell, J., Noachtar, S., and Brown, P. (2000). Organization of cortical activities related to movement in humans. J. Neurosci. 20, 2307-2314.

Marteniuk, R. G., and MacKenzie, C. L. (1980). Information processing in movement organization and execution. In Attention and performance VIII, R. Nickerson, ed. (Hillsdale, NJ, Erlbaum), pp. 29-57.

Matteis, M., Vernieri, F., Troisi, E., Pasqualetti, P. Tibuzzi, F., Caltagirone, C., and Silvestrini, M. (2003). Early cerebral hemodynamic changes during passive movements and motor recovery after stroke. J. Neurol. 250, 810-817.

Mechelli, A., Price, C. J., Noppeney, U., and Friston, K. J. (2003). A dynamic causa modeling study on category effects: bottom-up or top-down mediation? J.Cogn Neurosci. 15, 925-934.

Mima, T., Sadato, N., Yazawa, S., Hanakawa, T., Fukuyama, H., Yonekura, Y., and Shibasaki, H. (1999). Brain structures related to active and passive finger movements in man. Brain 122, 1989-1997.

Morgan, V. L., and Price, R. R. (2004). The effect of sensorimotor activation on functional connectivity mapping with MRI. Magn. Reson. Imaging 22, 1069-1075.

Nir, Y., Hasson, U., Levy, I., Yeshurun, Y., and Malach, R. (2006). Widespread functional connectivity and fMRI fluctuations in human visual cortex in the absence of visual stimulation. Neuroimage $30,1313-1324$.

Pashler, H. (1994). Dual-task interference in simple tasks: data and theory. Psychol. Bull. 116, 220-244.

Peltier, S. J., LaConte, S. M., Niyazov, D. M., Liu, J. Z., Sahgal, V., Yue, G. H., and Hu, X. P. (2005). Reductions in interhemispheric motor cortex functional connectivity after muscle fatigue. Brain Res. 1057, 10-16.

Penny, W. D., Stephan, K. E., Mechelli, A., and Friston, K. J. (2004). Modelling functional integration: a comparison of structural equation and dynamic causal models. Neuroimage 23, 264-274.

Pollok, B., Butz, M., Gross, J., and Schnitzler, A. (2007). Intercerebellar coupling contributes to bimanual coordination. J. Cogn. Neurosci. 19, 704-719.

Raichle, M. E., MacLeod, A. M., Snyder, A. Z., Powers, W. J., Gusnard, D. A., and Shulman, G. L. (2001). A default mode of brain function. Proc. Natl. Acad. Sci. USA 98, 676-682.

Rao, S. M., Binder, J. R., Bandettini, P. A., Hammeke, T. A., Yetkin, F. Z., Jesmanowicz, A., Lisk, L. M., Morris, G. L., Mueller, W. M., and Estkowski, L. D. (1993). Functiona magnetic resonance imaging of complex human movements. Neurology 43, 23112318.

Reddy, H., Floyer, A., Donaghy, M., and Matthews, P. M. (2001). Altered cortical activation with finger movement after peripheral denervation: comparison of active and passive tasks. Exp. Brain Res. 138, 484-491.

Rissman, J., Gazzaley, A., and D'Esposito, M. (2004). Measuring functional connectivity during distinct stages of a cognitive task. Neuroimage 23, 752-763.

Rokni, U., Steinberg, O., Vaadia, E., and Sompolinsky, H. (2003). Cortical representation of bimanual movements. J. Neurosci. 23, 11577-11586.

Rowe, J., Friston, K., Frackowiak, R., and Passingham, R. (2002). Attention to action: specific modulation of corticocortical interactions in humans. Neuroimage 17, 988998.

Ruben, J., Schwiemann, J., Deuchert, M., Meyer, R., Krause, T., Curio, G., Villringer, K. Kurth, R., and Villringer, A. (2001). Somatotopic organization of human secondary somatosensory cortex. Cereb. Cortex 11, 463-473.

Sadato, N., Yonekura, Y., Waki, A., Yamada, H., and Ishii, Y. (1997). Role of the supplementary motor area and the right premotor cortex in the coordination of bimanual finger movements. J. Neurosci. 17, 9667-9674.

Sahyoun, C., Floyer-Lea, A., Johansen-Berg, H., and Matthews, P. M. (2004). Towards an understanding of gait control: brain activation during the anticipation, preparation and execution of foot movements. Neuroimage 21, 568-575.

Schmidt, R. A. (1975). A schema theory of discrete motor skill learning. Psychol. Rev. 82 , 225-260.

Serrien, D. J., and Brown, P. (2004). Changes in functional coupling patterns during bimanual task performance. Neuroreport 15, 1387-1390.

Serrien, D. J., Nirkko, A. C., Lovblad, K. 0., and Wiesendanger, M. (2001). Damage to the parietal lobe impairs bimanual coordination. Neuroreport 12, 2721-2724.

Serrien, D. J., Ivry, R. B., and Swinnen, S. P. (2006). Dynamics of hemispheric specialization and integration in the context of motor control. Nat. Rev. Neurosci. 7 , 160-166.

Simoes, C., and Hari, R. (1999). Relationship between responses to contra- and ipsilateral stimuli in the human second somatosensory cortex SII. Neuroimage 10, 408-416.

Solodkin, A., Hlustik, P., Chen, E. E., and Small, S. L. (2004). Fine modulation in network activation during motor execution and motor imagery. Cereb. Cortex 14 , 1246-1255.

Stancak, A., Jr., PfurtschellerG. (1996). Event-related desynchronisation of central betarhythms during brisk and slow self-paced finger movements of dominant and nondominant hand. Cogn. Brain Res. 4, 171-183.

Sun, F. T., Miller, L. M., and D'Esposito, M. (2004). Measuring interregional functional connectivity using coherence and partial coherence analyses of fMRI data. Neuroimage 21, 647-658. 
Swinnen, S. P. Young, D. E., Walter, C. B., and Serrien, D. J. (1991). Control of asymmetrical bimanual movements. Exp. Brain Res. 85, 163-173.

Swinnen, S. P., Jardin, K. Meulenbroek, R., Dounskaia, N., and Hofkens-van den Brandt, M. (1997). Egocentric and allocentric constraints in the expression of patterns of interlimb coordination. J. Cogn. Neurosci. 9, 348-377.

Thickbroom, G. W., Byrnes, M. L., and Mastaglia, F. L. (2003). Dual representation of the hand in the cerebellum: activation with voluntary and passive finger movement. Neuroimage 18, 670-674.

Ullen, F., Forssberg, H., and Ehrsson, H. H. (2003). Neural networks for the coordination of the hands in time. J. Neurophysiol. 89, 1126-1135.

Varela, F., Lachaux, J. P., Rodriguez, E., and Martinerie, J. (2001). The brainweb: phase synchronization and large-scale integration. Nat. Rev. Neurosci. 2, 229-239.
Weiller, C J Juptner, M., Fellows, S., Riinties, M. Leonhardt, G., Kiebel, S., Muller, S., Diener, H. C., and Thilmann, A. F. (1996). Brain representation of active and passive movements. Neuroimage 4, 105-110.

Wenderoth, N., Debaere, F., Sunaert, S., Van, H. P., and Swinnen, S. P. (2004). Parietopremotor areas mediate directional interference during bimanual movements. Cereb. Cortex 14, 1153-1163.

Worsley, K. J Marrett, S., Neelin, P. Vandal, A. C. Friston, K. J., and Evans, A. C. (1996). A unified statistical approach for determining significant signals in images of cerebral activation. Hum. Brain Mapp. 4, 58-73.

Zhuang, J., LaConte, S., Peltier, S., Zhang, K., and Hu, X. (2005). Connectivity exploration with structural equation modeling: an $\mathrm{fMRI}$ study of bimanual motor coordination. Neuroimage 25, 462-470. 